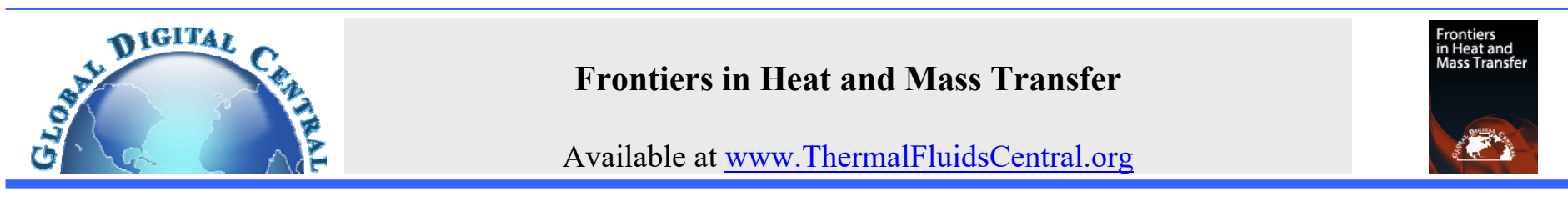

\title{
CONDUCTION, CONVECTION, AND RADIATION IN A CLOSED CAVITY WITH A LOCAL RADIANT HEATER
}

\author{
Geniy V. Kuznetsov, Alexander E. Nee* \\ National Research Tomsk Polytechnic University, Tomsk, 634050, Russia
}

\begin{abstract}
This study deals with the numerical investigation of combined heat transfer via conduction, laminar natural convection, and surface radiation in a closed rectangular cavity with a radiant heating source. Unsteady two-dimensional equations of mass, momentum, and energy conservation for complex heat transfer process under study were formulated in terms of the vorticity - stream function - temperature variables and solved by means of the finite difference method on a uniform grid. Developed numerical code was validated against two benchmark problems of convective and convective-radiative heat transfer. When analyzing complex heat transfer regularities, we varied the following parameters: dimensionless time $5 \leq \tau \leq 800$, Rayleigh number $6 \cdot 10^{4} \leq R a \leq 4 \cdot 10^{6}$, conduction-radiation number $19.71 \leq N_{r} \leq 78.84$, surface emissivity $0.2 \leq \varepsilon \leq 0.9$, emitter length $0.1 \leq D \leq 1$, walls thickness $0.05 \leq M \leq 0.25$. According to the results of mathematical modelling, it was found that the radiant parameters significantly affected the formation of differential and integral characteristics of conjugate heat transfer. Along with that the convective Nusselt number was slightly changed in geometrical and physical conditions under study. An increase in the walls thickness led to a reduce in the temperature of the air cavity. However, the effect of wall thickness on the convective and radiative Nusselt numbers was not significant.
\end{abstract}

Keywords: radiant heating, conjugate heat transfer, surface radiation, numerical simulation, finite difference method..

\section{INTRODUCTION}

Combined heat transfer by convection and radiation are encountered in many engineering systems. The most obvious examples are electronic equipment cooling systems, solar collectors, and building thermophysics. At the present time, complex heat transfer problems were numerically investigated in closed (Baek and Kim, 2002; Zhu and Yang, 2003; Bae and Hyun, 2004; Ait-taleb et. al., 2008; Anil Kumar Sharma et. al., 2008; Xin et. al., 2013; Cherifi et. al., 2015) and open (Nouanegue et. al., 2008; Mezrhab et. al., 2010; Rajkumar et. al., 2011; Hinojosa et. al., 2017) cavities under conditions of the laminar and turbulent flow regimes. However, in most cases the effect of heatconducting finite thickness walls on thermal regime of a domain was not taken into account when formulating boundary value problems of convective-radiative heat transfer. At the same time G.V. Kuznetsov and M.A. Sheremet (Kuznetsov and Sheremet, 2009; 2010; 2011) revealed that heat losses to the heat-accumulating finite thickness walls drastically changed both differential and integral heat transfer characteristics. The most relevant studies of combined heat transfer via natural convection and thermal surface radiation is presented below.

Analysis of convective-radiative heat transfer in domains filled with radiatively non-participating medium (Asako and Nakamura, 1982; Akiyama and Chong, 1997, Bouafia et. al., 2015) is generally carried out for differentially heated square cavities. The study of Asako and Nakamura (1982) is one of the first investigations of combined heat transfer via surface radiation and natural convection. In order to solve the equations of mass, momentum, and energy conservation, the authors used the finite difference method. M. Akiyama and Q.P. Chong (1997) performed numerical analysis for similar boundary value problem of complex heat transfer in a closed cavity with two vertical isothermal and two horizontal adiabatic walls. Steady equations of natural convection were solved by means of the finite volume method on a uniform grid $(42 \times 42)$ using SIMPLE algorithm. The authors assumed that working fluid satisfied Boussinesq approximation. To calculate the radiative heat exchange between gray diffusive surfaces, the netradiation method was used. According to the results of mathematical modelling, it was found that taking into account the surface radiation even with a small temperature difference $(\Delta T=29.35 K)$ led to an alteration of isotherms and fluid flow pattern in the square cavity. However, convective Nusselt number weakly depended on the surface emissivity in this case. The study of M. Bouafia et. al. (2015) is a further development of the work of Akiyama and Chong (1997). M. Bouafia et. al. (2015) conducted a numerical analysis of natural convection and surface radiation interaction in a differentially heated square cavity. The authors investigated the cases with strong nonBoussinesq effects. Control volume method and non-uniform staggered grid $257 \times 257$ were used to solve the boundary value problem. The radiosity equation was solved by means of the Gauss elimination method. According to the results of numerical modelling, it was found that the similar regularities observed by Wang et. al. (2006) for coupled natural convection and surface radiation problem in the cavity with perfectly conducting horizontal walls was not revealed when considering the non-Boussinesq regime. Whereas investigations (Asako and Nakamura, 1982; Akiyama and Chong, 1997, Bouafia et. al., 2015; Wang et. al., 2006) were carried out for domains with vertically oriented heating sources, M. Ashish Gad and C. Balaji (2010) analyzed convective-radiative heat transfer in a cavity heated from below. The governing equations were solved in FLUENT 6.3. Correlations for convective and radiative Nusselt numbers were obtained for the following range of parameters: $5 \cdot 10^{3} \leq R a \leq 10^{5}, \quad 1 \leq A R \leq 5$, $0.1 \leq \varepsilon \leq 0.85$.

The above studies were focused on the analysis of convectiveradiative heat transfer in cavities bounded by infinitely thin walls. However, this investigation deals with the numerical simulation of 
combined heat transfer via conduction in the finite thickness walls, laminar natural convection of the air, and thermal surface radiation of the solid-fluid interfaces. The closest related works on the subject of this study are investigations of Martyushev and Sheremet (2014a; 2014b). M.A. Sheremet and S.G. Martyushev (2014a) numerically analyzed the combined problem of natural convection and surface radiation in a closed square cavity filled with diathermic medium and bounded by heat-conducting finite thickness walls. A local isothermal heat-release source was fixed at the bottom horizontal wall. It was found that variation of the surface emissivity of the solid - fluid interfaces in a range of $0.1 \leq \varepsilon \leq 0.9$ did not lead to an alteration of flow pattern in the geometrical and physical conditions under study. Along with that the shape of isotherms was slightly changed. A reasonable conclusion could be made that the cavity was predominantly heated due to the natural convection mechanism of heat transfer. On the other hand, the aim of this study is to analyze the interaction of conduction, natural convection, and surface radiation in a closed rectangular cavity heated by a radiant energy source in a wide range of governing parameters. First of all, the geometrical and physical conditions under consideration are encountered in such engineering application as small-scale infrared chambers (Rasool Sadin et. al., 2014) for drying of different materials. Additionally, the results of numerical modelling can be applied when designing passive cooling systems for energy-saturated electronic components.

\section{PROBLEM FORMULATION}

\subsection{Geometrical and physical models}

A rectangular cavity filled with radiatively non-participating medium (air) and bounded by heat-conducting finite thickness walls with radiant energy source fixed at the top horizontal fluid - solid interface was considered. Figure 1 shows the solution domain of the boundary value problem under study.

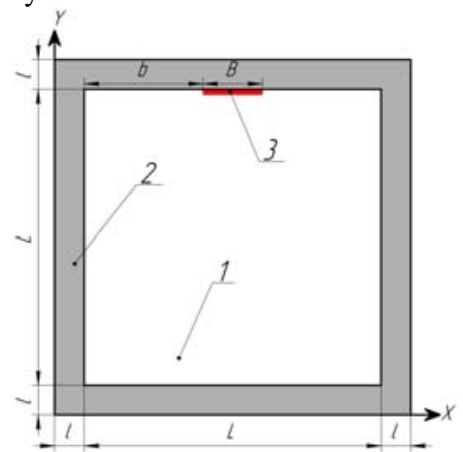

Fig. 1 Solution domain: 1 - air, 2 - enclosure, 3 - radiant energy source

When formulating the problem, it was assumed that the thermophysical properties of air, enclosure, and radiant energy source were temperature-independent. Unsteady two-dimensional laminar flow of viscous incompressible fluid was considered. External boundaries of the solution domain were assumed heat insulated. Equalities of heat fluxes and temperatures were set at the gray diffusive fluid - solid interfaces. It was assumed that the radiant energy source had already reached the stationary mode of operation at the initial moment of time. Therefore, the emitter surfaces were isothermal.

It was assumed that the fluid under consideration satisfied the Boussinesq approximation. It is well known (Suslov et. al., 1997; Codina, 2001; Vierendeels et. al., 2004) that this approximation is absolutely valid for small temperature differences $\left(\frac{T_{\max }-T_{\min }}{T_{\min }}=B u \leq 0.1\right)$ when solving problems of natural convection.

However, based on a numerical study, Zhong et. al. (1985) obtained correlation establishing the limits of application of the Boussinesq approximation. In accordance with (Zhong et. al., 1985), correlation
$B u=0.0244 \cdot R a^{0.243}$ makes it possible to determine the limiting Boussinesq number within the error in the calculated heat transfer characteristics does not exceed 5\%. Moreover, Hamimid et. al. (2016) performed a comparative analysis between an incompressible code based on the Boussinesq approximation and the low Mach number compressible code with temperature-dependent coefficients. Based on the results of numerical investigation, it was found that the deviation of the temperatures at $R a=10^{6}$ and $\Delta T=360 \mathrm{~K}$ did not exceed $4 \%$. Therefore, in this study, the Boussinesq number was altered in a range of $0.35 \leq B u \leq 0.98$ in accordance with a variation of the Rayleigh number from $6 \cdot 10^{4}$ to $4 \cdot 10^{6}$. Boussinesq number was equal to 0.51 for temperature difference under consideration.

\subsection{Mathematical model}

Neglecting viscous dissipation of energy, Navier-Stokes and energy equations under Boussinesq approximation in the vorticity - stream function - temperature dimensionless variables are as follows (Kuznetsov and Sheremet, 2009; Martyushev and Sheremet, 2014b):

$$
\begin{gathered}
\frac{\partial \Omega}{\partial \tau}+\frac{\partial \Psi}{\partial Y} \frac{\partial \Omega}{\partial X}-\frac{\partial \Psi}{\partial X} \frac{\partial \Omega}{\partial Y}=\sqrt{\frac{\operatorname{Pr}}{\mathrm{Ra}}} \cdot\left(\frac{\partial^{2} \Omega}{\partial X^{2}}+\frac{\partial^{2} \Omega}{\partial Y^{2}}\right)+\frac{\partial \Theta_{1}}{\partial X}, \\
\frac{\partial^{2} \Psi}{\partial X^{2}}+\frac{\partial^{2} \Psi}{\partial Y^{2}}=-\Omega, \\
\frac{\partial \Theta_{1}}{\partial \tau}+\frac{\partial \Psi}{\partial Y} \frac{\partial \Theta_{1}}{\partial X}-\frac{\partial \Psi}{\partial X} \frac{\partial \Theta_{1}}{\partial Y}=\frac{1}{\sqrt{\mathrm{Ra} \cdot \operatorname{Pr}}} \cdot\left(\frac{\partial^{2} \Theta_{1}}{\partial X^{2}}+\frac{\partial^{2} \Theta_{1}}{\partial Y^{2}}\right), \\
\frac{\partial \Theta_{2}}{\partial \tau}=F O_{2}\left(\frac{\partial^{2} \Theta_{2}}{\partial X^{2}}+\frac{\partial^{2} \Theta_{2}}{\partial Y^{2}}\right), \\
\frac{\partial \Theta_{3}}{\partial \tau}=F O_{3}\left(\frac{\partial^{2} \Theta_{3}}{\partial X^{2}}+\frac{\partial^{2} \Theta_{3}}{\partial Y^{2}}\right) .
\end{gathered}
$$

Initial conditions for equations (1) - (5) are as follows:

$$
\begin{gathered}
\Psi(X, Y, 0)=0 ; \Omega(X, Y, 0)=0, \\
\Theta_{1}(X, Y, 0)=\Theta_{2}(X, Y, 0)=\Theta_{3}(X, Y, 0)=0 .
\end{gathered}
$$

Boundary conditions for equations $(1)-(5)$ are as follows: at the surface of the emitter:

$$
\Theta_{3}(X, Y, \tau)=1 .
$$

at the external boundaries of the solution domain:

$$
\frac{\partial \Theta_{2}(X, Y, \tau)}{\partial n}=0 .
$$

at the interface of $\mathrm{Y}=1,1<\mathrm{X}<\mathrm{L}+\mathrm{l}$ :

$$
\Psi=0, \frac{\partial \Psi}{\partial Y}=0, \Omega=-\frac{\partial^{2} \Psi}{\partial Y^{2}},\left\{\begin{array}{l}
\Theta_{2}=\Theta_{1}, \\
\frac{\partial \Theta_{2}}{\partial Y}=\frac{\lambda_{1}}{\lambda_{2}} \cdot \frac{\partial \Theta_{1}}{\partial Y}+N_{r} \cdot Q_{r} .
\end{array}\right.
$$

at the interfaces of $\mathrm{Y}=\mathrm{l}+\mathrm{L}, \mathrm{l}<\mathrm{X}<\mathrm{b}+\mathrm{l}, 1+\mathrm{b}+\mathrm{B}<\mathrm{X}<\mathrm{l}+\mathrm{L}$ :

$$
\Psi=0, \frac{\partial \Psi}{\partial Y}=0, \Omega=-\frac{\partial^{2} \Psi}{\partial Y^{2}},\left\{\begin{array}{l}
\Theta_{1}=\Theta_{2}, \\
\frac{\partial \Theta_{1}}{\partial Y}=\frac{\lambda_{2}}{\lambda_{1}} \cdot \frac{\partial \Theta_{2}}{\partial Y}+N_{r} \cdot Q_{r} .
\end{array}\right.
$$

At the interface of $\mathrm{X}=1,1<\mathrm{Y}<\mathrm{L}+1$ :

$$
\Psi=0, \frac{\partial \Psi}{\partial X}=0, \Omega=-\frac{\partial^{2} \Psi}{\partial X^{2}},\left\{\begin{array}{l}
\Theta_{2}=\Theta_{1}, \\
\frac{\partial \Theta_{2}}{\partial X}=\frac{\lambda_{1}}{\lambda_{2}} \cdot \frac{\partial \Theta_{1}}{\partial X}+N_{r} \cdot Q_{r} .
\end{array}\right.
$$

At the interface of $\mathrm{X}=1+\mathrm{L}, 1<\mathrm{Y}<\mathrm{L}+\mathrm{l}$ :

$$
\Psi=0, \frac{\partial \Psi}{\partial X}=0, \Omega=-\frac{\partial^{2} \Psi}{\partial X^{2}},\left\{\begin{array}{l}
\Theta_{1}=\Theta_{2}, \\
\frac{\partial \Theta_{1}}{\partial X}=\frac{\lambda_{2}}{\lambda_{1}} \cdot \frac{\partial \Theta_{2}}{\partial X}+N_{r} \cdot Q_{r} .
\end{array}\right.
$$

To reduce the system of equations to its dimensionless form, the following relations were used: 


$$
\begin{gathered}
X=\frac{x}{L} ; \quad Y=\frac{y}{L} ; \quad D=\frac{B}{L} ; \quad M=\frac{l}{L} ; \quad \tau=\frac{t}{t_{0}} ; \quad U=\frac{u}{V_{n c}} ; \quad V=\frac{v}{V_{n c}} ; \\
\Theta=\frac{T-T_{0}}{T_{h}-T_{0}} ; \Psi=\frac{\psi}{\psi_{0}} ; \quad \Omega=\frac{\omega}{\omega_{0}} ; \quad V_{n c}=\sqrt{g \cdot \beta \cdot\left(T_{h}-T_{0}\right) \cdot L} ; t_{0}=\frac{L}{V_{n c}} ; \\
\psi_{0}=V_{n c} L ; \quad \omega_{0}=\frac{V_{n c}}{L} ;
\end{gathered}
$$

\subsection{Radiation model}

In order to calculate the radiant heat exchange in a cavity filled with radiatively non-participating medium, the net-radiation method was used. The solution domain was divided into $N$ surface elements, where radiative properties could be taken as constant. It was assumed that the fluid - solid interfaces were gray. Radiation reflected from any surface was diffusely distributed in the cavity. In this case, the entire incident radiation flux was reflected with the same intensity, regardless of the direction from which it came. The view factors were determined by means of the Hottel's crossed string method (Hottel and Sarofim, 1967). Taking into account the above assumptions, the non-dimensional radiosity equation can be written as follows (Bouafia et. al., 2015):

$$
J_{i}=\varepsilon_{i} \cdot\left[\Theta_{i} \cdot(1-\chi)+\chi\right]^{4}+\left(1-\varepsilon_{i}\right) \cdot \sum_{j=1}^{N} F_{i-j} \cdot J_{j},
$$

where $J_{i}$ is the non-dimensional radiosity from the $i$ element, $\varepsilon_{i} \cdot\left[\Theta_{i} \cdot(1-\chi)+\chi\right]^{4}-\quad$ is self-radiation of the $i$ element, $\left(1-\varepsilon_{i}\right) \cdot \sum_{j=1}^{N} F_{i-j} \cdot J_{j} \quad$ is reflected radiant energy coming from surrounding $j$ elements that can be seen by the $i$ element.

Calculating the values of the $J_{i}$, irradiation equation can be determined as follows (Bouafia et. al., 2015):

$$
Q_{r, i}=J_{i}-\sum_{j=1}^{N} F_{i-j} \cdot J_{j},
$$

\subsection{Heat transfer rate}

The mean Nusselt numbers characterized the heat transfer rate by natural convection $\left(N u_{c o n}\right)$ and thermal radiation $\left(N u_{\text {rad }}\right)$ can be calculated as follows (Xin et. al., 2013, Akiyama and Chong, 1997, Bouafia et. al., 2015):

$$
\begin{gathered}
N u_{c o n, F}=\int_{0.1}^{1.1}\left|\frac{\partial \Theta}{\partial X}\right|_{X=0.1} \cdot d Y, N u_{c o n, W}=\int_{0.1}^{1.1}\left|\frac{\partial \Theta}{\partial Y}\right|_{Y=0.1} \cdot d X, \\
N u_{r a d, F}=\int_{0.1}^{1.1} N_{r} \cdot Q_{r} \cdot d Y, N u_{r a d, W}=\int_{0.1}^{1.1} N_{r} \cdot Q_{r} \cdot d X .
\end{gathered}
$$

The total Nusselt number is determined as follows (Xin et. al., 2013, Akiyama and Chong, 1997, Bouafia et. al., 2015):

$$
N u_{t}=N u_{c o n}+N u_{r a d}
$$

\subsection{Numerical procedure}

The system of unsteady two-dimensional partial differential equations (1) - (5) with the corresponding initial (6), (7) and boundary (8) - (13) conditions was numerically solved by means of the finite difference method (Kuznetsov and Sheremet, 2009; 2010; 2011). To approximate the equations (1) - (5), the implicit four-point pattern and the second order schemes of accuracy (Roache, 1976) were used. Convective terms were approximated by the monotonic scheme of Samarskii (Samarskii, 1977). Diffusive terms were discretized by the central differences (Samarskii, 1977). Radiosity equations (14) were solved by means of the Gauss elimination method (Bouafia et. al., 2015). The boundary conditions (9) - (11) were approximated by schemes of the second order of accuracy. To determine the vorticity at the fluid - solid interfaces, we used Wood's formula (Roache, 1976).

\subsection{Validation}

Developed numerical code was validated against three benchmark problems of heat transfer (de Vahl Davis, 1983; Akiyama and Chong, 1997; Aydin, 1999). At the first stage, a classical problem of natural convection in a closed square cavity with two vertical isothermal and two horizontal adiabatic walls was solved. Calculations were performed on a uniform grid $257 \times 257$. Numerical investigations were conducted in a range of Rayleigh number $10^{3} \leq R a \leq 10^{6}$ when Prandtl number was $\operatorname{Pr}=0.71$. Obtained mean Nusselt numbers were compared with the published data (de Vahl Davis, 1983; Dixit and Babu, 2006; Markatos and Pericleous, 1984, Jani et. al., 2014) and given in Table 1.

Table 1. Comparison of the mean Nusselt numbers

\begin{tabular}{|c|c|c|c|c|c|}
\hline $\mathrm{Ra}$ & $\begin{array}{c}N u_{a v} \\
(\text { de Vahl } \\
\text { Davis, } \\
1983)\end{array}$ & $\begin{array}{c}N u_{a v} \\
\text { (Dixit } \\
\text { and } \\
\text { Babu, } \\
2006)\end{array}$ & $\begin{array}{c}N u_{a v} \\
\text { (Markatos } \\
\text { and } \\
\text { Pericleous, } \\
1984)\end{array}$ & $\begin{array}{c}N u_{a v} \\
\text { (Jani } \text { et. } \\
\text { al. }\end{array}$ & $\begin{array}{c}\text { This } \\
\text { study }\end{array}$ \\
\hline $10^{3}$ & 1.118 & 1.121 & 1.108 & 1.113 & \\
\hline $10^{4}$ & 2.243 & 2.286 & 2.201 & 2.254 & 2.213 \\
\hline $10^{5}$ & 4.519 & 4.5463 & 4.43 & 4.507 & 4.515 \\
\hline $10^{6}$ & 8.799 & 8.652 & 8.754 & 8.802 & 8.897 \\
\hline
\end{tabular}

At the second stage, combined problem of natural convection and surface radiation was solved for a differentially heated cavity. Numerical simulation was performed for similar geometrical and physical conditions analyzed by M. Akiyama and Q. P. Chong (1997). Isotherms for different Rayleigh numbers are shown in Figure 2.
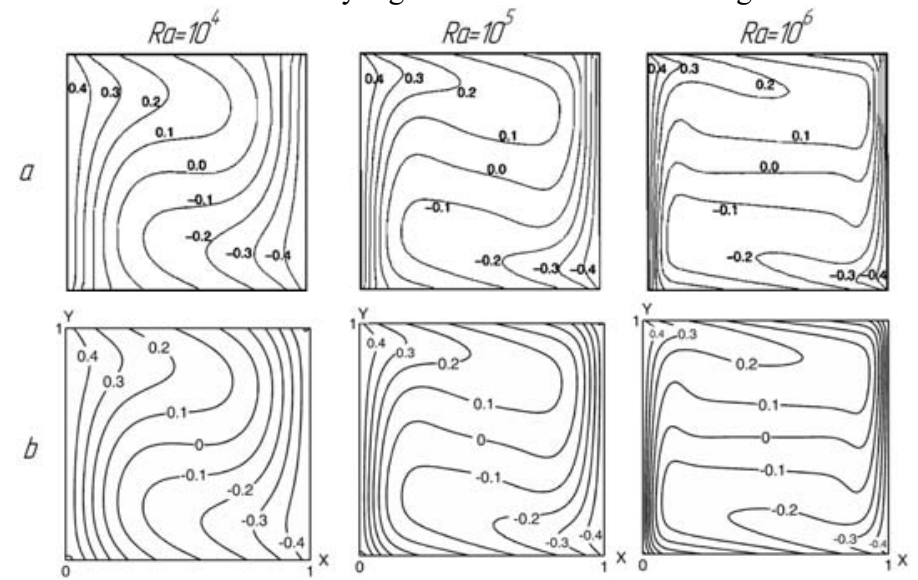

Fig. 2 Isotherms for different Rayleigh numbers: $a-\mathrm{M}$. Akiyama and Q. P. Chong (1997), $b$ - this study.

At the last stage, a problem of the two-dimensional transient natural convection of single-phase fluid inside a completely filled square enclosure was solved for Prandtl number of 0.71 and Rayleigh number of $10^{6}$. Isotherms and streamlines are presented in Fig. 3.

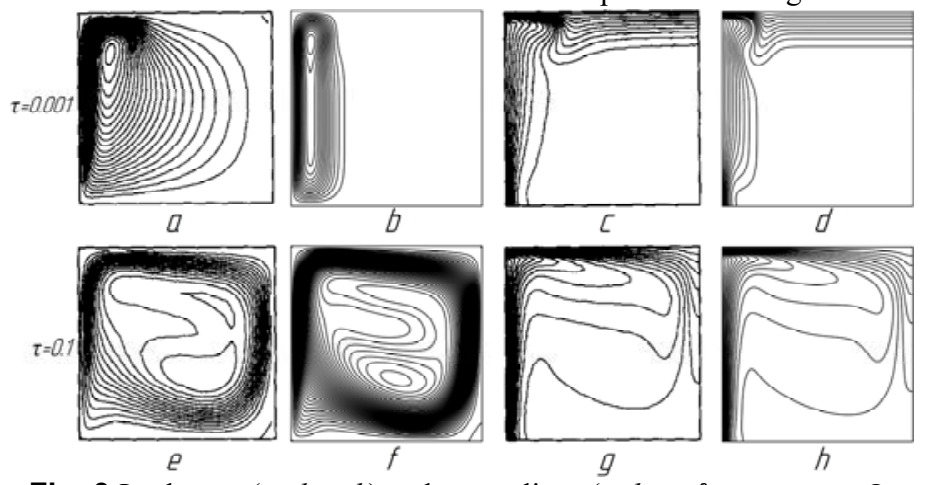

Fig. 3 Isotherms $(c, d, g, h)$ and streamlines $(a, b, e, f): a, c, e, g-\mathrm{O}$. Aydin (1999), $b, d, f, h$ - this study. 
The presented comparisons of differential and integral heat transfer characteristics clearly demonstrated the adequacy of the developed numerical code.

\subsection{Grid dependence}

In order to choose the optimal grid size, numerical code was tested at the several grid points: 51x51, 101x101, 151x151, 201x201. According to the results of the mathematical modelling (not presented in this paper), it was found that the deviation of temperatures was less than $1 \%$ when grid size was $151 \times 151$ and $201 \times 201$. Thus, calculations were performed on a uniform grid $151 \times 151$

\section{RESULTS AND DISCUSSION}

Numerical investigation of combined heat transfer via conduction, laminar natural convection, and surface radiation were carried out for the cavity filled with air $(\mathrm{Pr}=0.71)$, whereas this working fluid is typical for many engineering systems. Temperature difference was constant $\Delta T=150 K$. The Rayleigh number was varied in a range of $6 \cdot 10^{4} \leq R a \leq 4 \cdot 10^{6}$, which was corresponded to a change in the characteristic size of the cavity from $0.025 \mathrm{~m}$ to $0.1 \mathrm{~m}$. The conductionradiation number was set $N_{r}=78.84$. Surface emissivity $\varepsilon$ was the same for all fluid-solid interfaces and changed from 0.2 to 0.9 . Initial temperature of the solution domain was $T_{0}=293 \mathrm{~K}$. Surface temperature of the radiant heating source was $T_{h}=443 \mathrm{~K}$. Positive values of streamlines represent clockwise circulation and negative values represent counterclockwise circulation.

\subsection{Effect of time}

The dynamics of the complex heat transfer process under study is quite clearly observed on the isotherms and streamlines shown in Fig. 4 for different values of the dimensionless time.
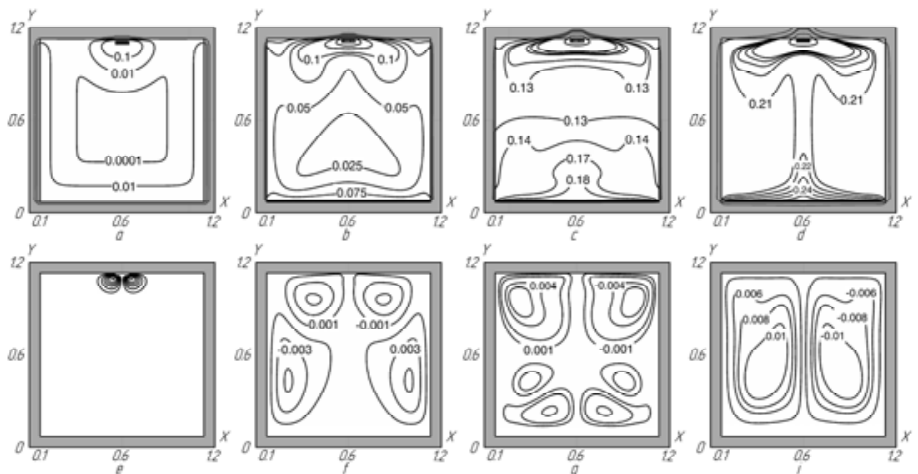

Fig. 4 Isotherms $(a, b, c, d)$ and streamlines $(e, f, g, i)$ when

$R a=4 \cdot 10^{6}, N_{r}=78.84, D=0.1$ and $\left.\left.\varepsilon=0.9: a, e\right) \tau=5 ; b, f\right) \tau=50$; $c$, g) $\tau=300 ; d, i) \tau=600$.

Presented characteristics illustrate significant unsteady nature of the conjugate heat transfer process under study. When $\tau=5$ (Fig. $4 a$, $e$ ), small circulating flows were formed near the radiant heating source, which was associated with a high temperature gradient near the emitter. The fluid-solid interfaces were uniformly heated due to the radiation flux coming from the radiant energy source. An increase in the dimensionless time to 50 (Fig. $4 b, f$ ) led to a rise in the size of convective cells near the radiant heating source. Air as absolutely transparent medium for thermal radiation was heated only near the fluid-solid interfaces due to the natural convection. As a result, circulating flows were formed in the bottom half of the cavity. When $\tau=300$ (Fig. $4 c, g$ ), a convective plume was created near the bottom horizontal fluid-solid interface. This factor led to a breakdown of the circulating flows in the bottom half of the cavity into four convective cells. The isotherm $\Theta=0.1$ monotonously moved to the vertical walls due to the air conduction near the radiant heating source. Along with that convective cells were increased in the size near the emitter. Further increase in the dimensionless time to 600 (Fig. $4 d$, $i$ ) led to a formation of the convective torch in the medium plane of the solution domain. The heated air near the bottom horizontal fluid-solid interface was ascended due to the natural convection mechanism of heat transfer. Then, the air was cooled due to the heat losses to the walls and descended along the vertical fluid-solid interfaces. As a result, only two large-scale convective cells were formed in the solution domain.

Figure 5 shows temperature profiles in the characteristic sections of the solution domain.
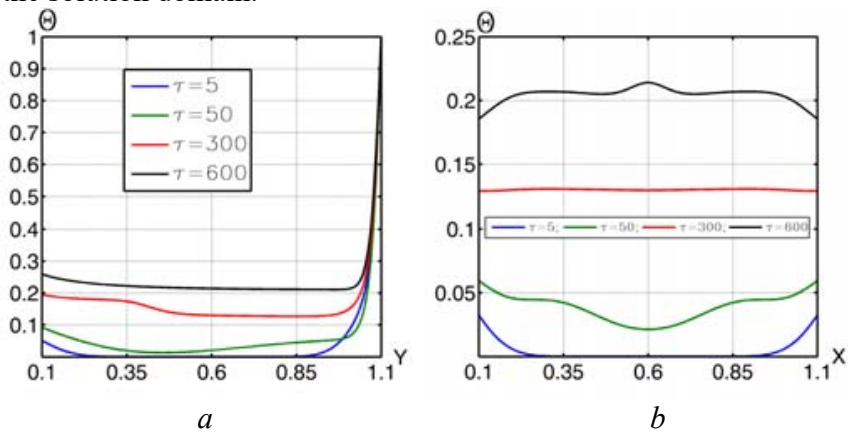

Fig. 5 Temperature profiles in the air cavity when $R a=4 \cdot 10^{6}$, $N_{r}=78.84, D=0.1$ and $\varepsilon=0.9$ in the sections of: $a-X=0.6 ; b-$ $Y=0.6$.

It was clearly seen that an increase in the time led to a rise in the temperature of the fluid-solid interfaces, which was obviously associated with the energy supply from the radiant heating source. As a result, vertical temperature gradient was reduced (Fig. 5 a). However, the temperature was slightly changed near the radiant energy source due to the predominance of conduction in the area of $1.05<Y<1.1$ (Fig. $5 a$ ). In the section of $Y=0.6$ (Fig. $5 b$ ), the extremum of function $\Theta=f(X)$ was located in the medium plane due to the absence of the heat removal at the external boundaries of the vertical walls. The heated air was ascended from the bottom to the top horizontal fluid-solid interface. As a result, the temperature was increased in the section of $Y=0.6$ (Fig. $5 b$ ) with time.

\subsection{Effect of Rayleigh number}

Figure 6 presents isotherms and streamlines illustrated the effect of buoyancy force on formation of the differential heat transfer characteristics.
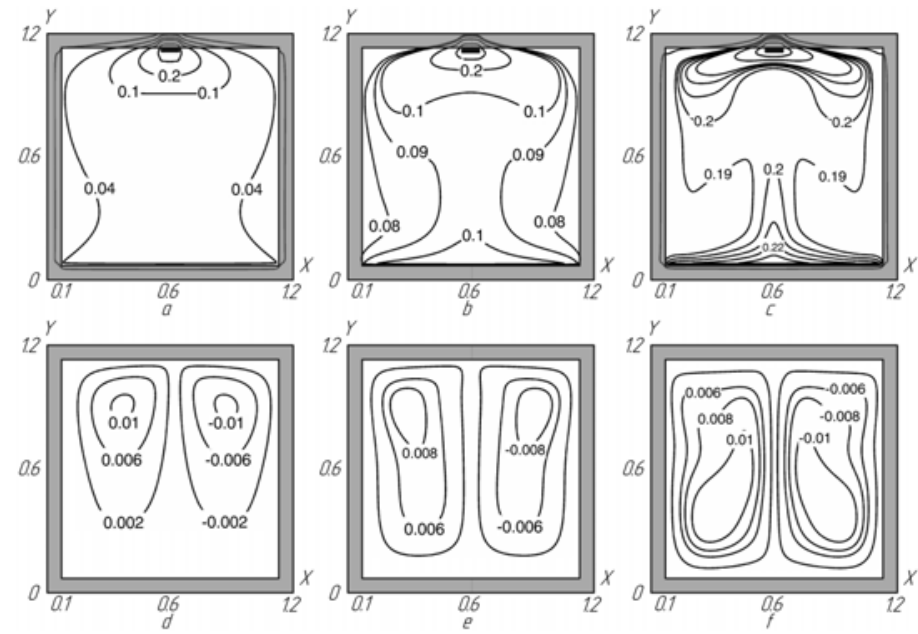

Fig. 6 Isotherms $(a, b, c)$ and streamlines $(e, f, g)$ when $\tau=500$ $D=0.1$ and $\left.\varepsilon=0.9: a, d) R a=6 \cdot 10^{4}, N_{r}=19.71 b, e\right) R a=5 \cdot 10^{5}$, $\left.N_{r}=40.6 ; c, f\right) R a=4 \cdot 10^{6}, N_{r}=78.84$.

It was clearly seen that an increase in the Rayleigh number led to a significant modification of heat transfer and fluid flow characteristics. However, it should be noted that only two large-scale convective cells were formed in the air cavity in all cases under study. Along with that the core of the convective cells was displaced from the top to the bottom horizontal fluid-solid interface, when the buoyancy force was increased. This factor was obviously associated with an increase in the air velocity due to an enhancement of the natural convection. When $R a \leq 5 \cdot 10^{5}$ (Fig. $6 a, b$ ), the air was slightly heated, which was 
associated with the low values of the conduction-radiation number. At the same time convective plumes were formed near the bottom horizontal fluid-solid interface. Further increase in the Rayleigh number to $4 \cdot 10^{6}$ (Fig. $6 \mathrm{c}$ ) led to a rise in the temperature of the solution domain. Along with that convective plumes were transformed into the convective torch. Figure 7 shows the temperature profiles in the characteristic sections of the air cavity.

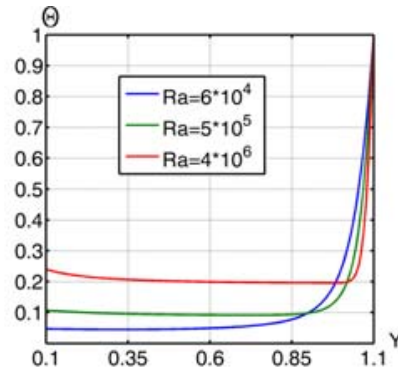

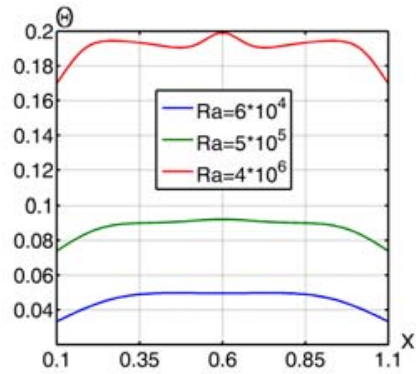

$b$

Fig. 7. Temperature profiles when $\tau=500, D=0.1$ and $\varepsilon=0.9$ in the sections of: $a-X=0.6 ; b-Y=0.6$.

It was clearly seen that an increase in the Rayleigh number in a range of $6 \cdot 10^{4} \leq R a \leq 4 \cdot 10^{6}$ led to a rise in the temperature of the fluid-solid interfaces approximately five times. Primarily, this factor was obviously associated with an increase in the conduction-radiation number. As a result, the values of the radiant fluxes coming to the fluidsolid interfaces were increased. Along with that a rise in the buoyancy force led to an increase in the temperature in the section of $Y=0.6$ (Fig. $7 \mathrm{~b}$ ) due to a more intensive motion of heated air from the bottom to the top horizontal wall.

\subsection{Effect of emissivity}

When analyzing the combined problems due to the natural convection and radiation in cavities filled with radiatively non-participating medium, the crucial parameter is the walls emissivity, whereas this factor determines radiation heat transfer rate. Figure 8 presents isotherms and streamlines illustrated the effect of the fluid-solid interfaces emissivity on differential heat transfer characteristics.
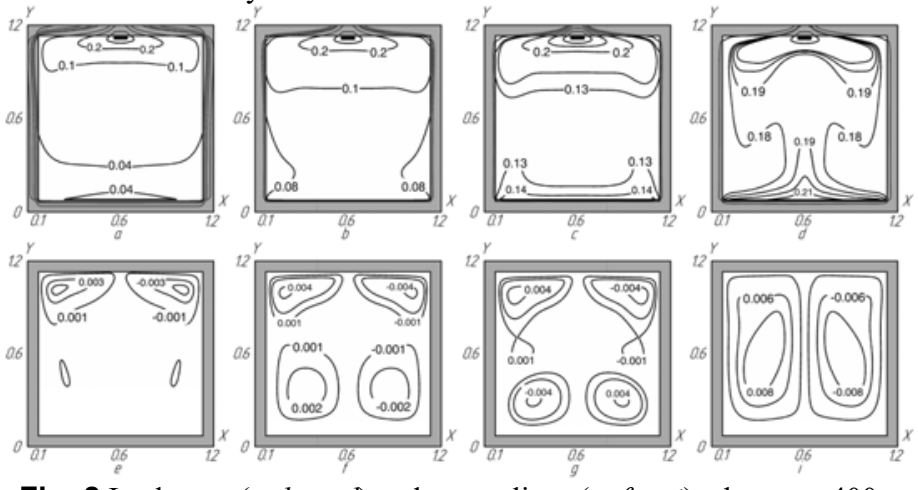

Fig. 8 Isotherms $(a, b, c, d)$ and streamlines $(e, f, g, i)$ when $\tau=400$, $R a=4 \cdot 10^{6}, D=0.1$ and $\left.\left.\left.N_{r}=78.84: a, e\right) \varepsilon=0.2 ; b, f\right) \varepsilon=0.4 ; c, g\right)$

$$
\varepsilon=0.6 ; d, i) \varepsilon=0.8 \text {. }
$$

Based on the analysis of the presented mathematical modelling results, a reasonable conclusion could be made that an increase in the fluid-solid interfaces emissivity led not only to a rise in the temperature, but fluid flow pattern was also changed. When $\varepsilon \leq 0.6$, we observed a thermal stratification of the air in the top half of the cavity (Fig. $8 a, b$, $c$ ), which was obviously associated with the predominance of conduction near the radiant heating source. Two convective cells were formed near the emitter and two circulating flows were created in the bottom half of the solution domain (Fig. $8 e, f, g$ ). When $\varepsilon=0.6$, convective plumes were formed near the bottom horizontal fluid-solid interface owing to temperature gradient increasing. It should be noted that this factor led to a change in the direction of circulating flows in the area of $0.1<Y<0.5,0.1<X<1.1$ (Fig. $8 \mathrm{~g}$ ) in comparison with the case when $\varepsilon=0.4$ (Fig. $8 \mathrm{f}$ ). Further increase in the emissivity of the fluidsolid interfaces to $\varepsilon=0.8$ led to a formation of a convective torch. As a result, the heated air was actively ascended at the medium plane of the solution domain. This factor led to a bend of the isotherms in the area of $0.8<\mathrm{Y}<1,0.1<\mathrm{X}<1.1$ (Fig. $8 d$ ). Hence, thermal stratification was disturbed in this area. Along with that circulating flows were merged into two large-scale convective cells. Figure 9 presents temperature profiles in the characteristic sections of the air cavity.
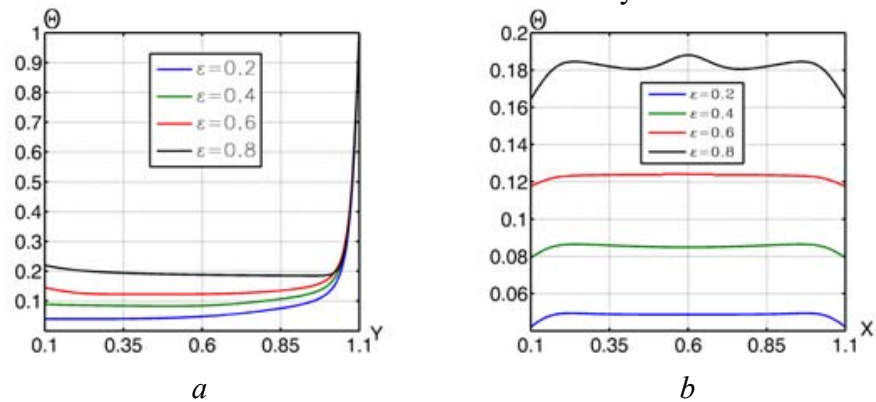

Fig. 9 Temperature profiles when $\tau=400, R a=4 \cdot 10^{6}, D=0.1$ and $N_{r}=78.84$ in the sections of: $a-X=0.6 ; b-Y=0.6$.

It was clearly seen that an increase in the walls emissivity four times led to a rise in the temperature of the fluid-solid interfaces 3.7 times. Obviously, when emissivity was increased, the interfaces started to absorb more radiant energy. As a result, the temperature rose. Along with that the air temperature was monotonously changed before the area of $1<\mathrm{Y}<1.1$ (Fig. $9 a$ ) near the radiant heating source. When $\varepsilon \leq 0.6$, a thermal stratification was clearly observed in the section of $Y=0.6$ (Fig. $9 \mathrm{~b}$ ). When $\varepsilon=0.8$, extremum of function $\Theta=f(X)$ (Fig. $9 \mathrm{~b}$ ) was located at the medium plane due to the convective torch formation.

\subsection{Effect of emitter length}

Isotherms and streamlines illustrated the effect of radiant energy source on heat transfer characteristics are presented in Fig. 10.
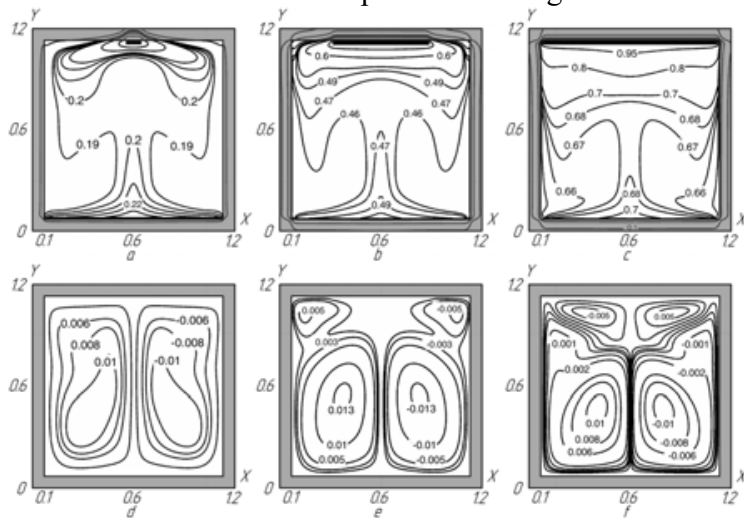

Fig. 10 Isotherms $(a, b, c)$ and streamlines $(d, e, f)$ when $\tau=500$, $R a=4 \cdot 10^{6}, \varepsilon=0.9$ and $\left.\left.N_{r}=78.84: a, d\right) D=0.1 ; b, e\right) D=0.55 ; c, f$ ) $D=1$.

As might be expected, an increase in the emitter length led to a rise in the absolute temperature of the solution domain (Fig. $10 a, b, c$ ). Heat transfer rate by the radiation was enhanced with growth of $D$. As a result, temperature of the solid-fluid interfaces was increased due to a rise in the radiant heat fluxes. Along with that flow pattern was altered in the top half of the cavity (Fig. $10 d, e, f$ ). Predominance of conduction near the radiant heating source led to a formation of secondary circulating currents and thermal stratification in the area of $0.8<\mathrm{Y}<1.1,0.1<\mathrm{X}<1.1$ when $D \geq 0.55$. An ascended flow of heated air was formed at the bottom horizontal solid-fluid interface in all variants considered (Fig. 10). With a relatively small length of the emitter $(D=0.1)$ convective plume reached the bottom wall of the radiant energy source, thereby thermal stratification in the area of $0.7<\mathrm{Y}<1$, 
$0.1<\mathrm{X}<1.1$ (Fig. $10 a$ ) was disarranged. With a growth of $D$ to $0.55 \mathrm{a}$ zone of gas heating due to the conduction near the emitter was increased. High temperature air in the area of $0.7<\mathrm{Y}<1,0.1<\mathrm{X}<1.1$ prevented a movement of ascending flow from the bottom to the top wall which was probably associated with the equalities of gas temperatures and, consequently, densities in this area. An analogous regularity was observed for $D=1$ (Fig. $10 c, f$ ). Moreover, a conventional boundary (Fig. $10 \mathrm{f}$ ) dividing the areas of gas heating due to the conduction near the emitter and circulating currents caused by natural convective heat transfer mechanism was clearly formed in this variant of numerical modelling. Figure 11 presents temperature profiles in the characteristic sections of the air cavity.
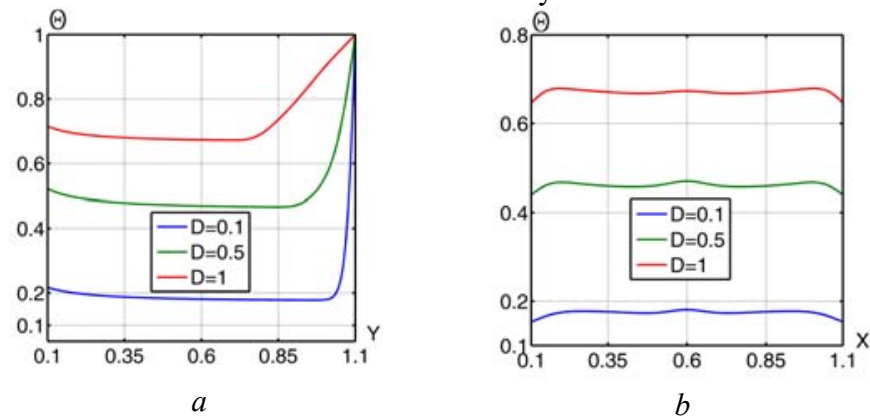

Fig. 11 Temperature profiles when $R a=4 \cdot 10^{6}, \tau=500, N_{r}=78.84$ and $\varepsilon=0.9$ in the sections of: $a-X=0.6 ; b-Y=0.6$.

It was clearly seen (Fig. $11 a$ ) that an increase in the radiant heating source length led to a reduce in the vertical temperature gradient. A reasonable conclusion could be made that the values of radiant fluxes coming to the solid-fluid interfaces were raised with a growth of $D$. This regularity was obviously associated with an enhancement of radiant heat exchange. However, it should be noted that an increase in the emitter length 10 times led to a rise in the temperature of the bottom horizontal solid-fluid interface (Fig. $11 a$ ) approximately 3.3 times. At the same time temperature of the vertical solid-fluid interface (Fig. $11 \mathrm{~b}$ ) was increased around 4.2 times. Along with that variation of the emitter length led to a change in the area of gas heating due to the conduction near the radiant energy source. When $D$ was equal to $0.1,0.5$ and 1 the area of gas heating due to the conduction was $1<Y<1.1,0.94<Y<1.1$ and $0.76<Y<1.1$ (Fig. 11 a), respectively.

\subsection{Effect of walls thickness}

In this subsection, the effect of the walls thickness bounded the gas cavity on the conductive-convective-radiative heat transfer characteristics is analyzed. The results of the numerical modelling are shown in Fig. 12.
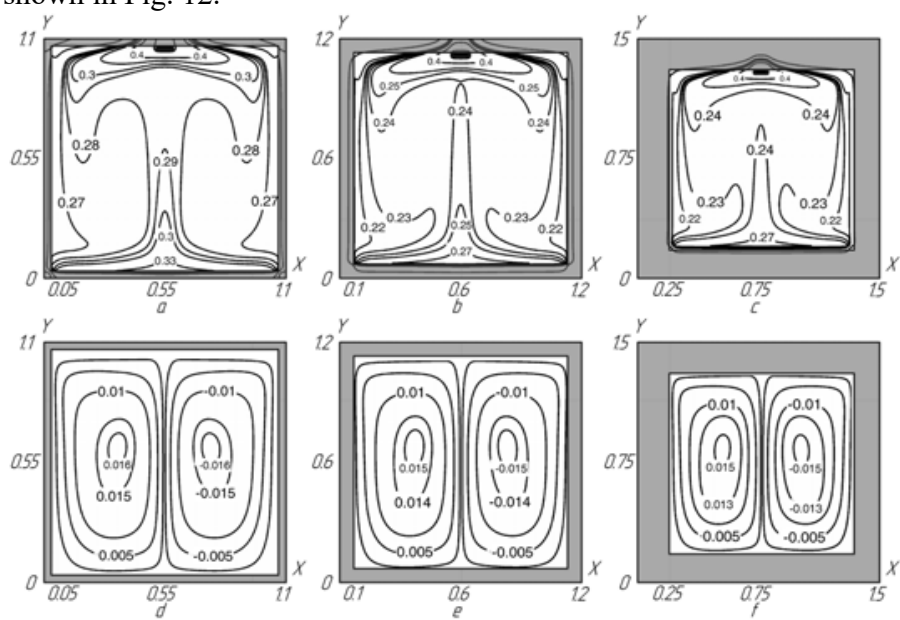

Fig. 12 Isotherms $(a, b, c, d)$ and streamlines $(e, f, g, i)$ when $\tau=800$, $\varepsilon=0.9, D=0.1, R a=4 \cdot 10^{6}$ and $\left.\left.N_{r}=78.84: a, d\right) M=0.05 ; b, e\right)$

$M=0.1 ; c, f) M=0.25$.
Primarily, an increase in the walls thickness led a reduce in the temperature of the air cavity (Fig. $12 a, b, c$ ). Obviously, amount of heat absorbed by finite thickness walls was increased with growth of $M$ and adiabatic boundary conditions at the external surfaces of the solution domain. Based on the heat balance equation, we could conclude that the more energy would be accumulated by walls the less energy would be accumulated by gas. As a result, air was cooled more intensive due to the heat losses to the enclosure with a growth of the walls thickness. However, it should be noted that the variation of $M$ from 0.1 to 0.25 led to a slightly change of the local characteristics of complex heat transfer in a range of governing parameters under consideration. It could be assumed that temperature gradient was the same within $\tau=800$ and $0.1 \leq M \leq 0.25$ (Fig. $12 b, c$ ). Along with that an identical flow pattern consisted of two large-scale convective cells was formed in all numerical modelling variants (Fig. $12 d, e, f$ ) under discussion.

\subsection{Heat transfer rate}

The major criterion characterized the heat transfer rate via convection and radiation is the mean Nusselt number. Figure 13 presents dependences of the mean Nusselt numbers versus dimensionless time, Rayleigh number, fluid-solid interfaces emissivity, emitter length, and walls thickness.

It was clearly seen that maximum values of the convective Nusselt numbers were reached both at the bottom horizontal (Fig. $13 a$ ) and vertical (Fig. $13 b$ ) fluid-solid interfaces when $\tau=1$. Probably, this factor was associated with the maximum temperature gradient between the interfaces and air boundary layer at the initial time. Hereafter, a gradual equalization of the temperature occurred near the fluid-solid interfaces, which led to a monotonic reduce of the $N u_{c o n}$. On the other hand, the radiative Nusselt number was slightly altered with time, which was obviously associated with the boundary conditions of the first kind set for radiant energy source. Along with that a reasonable conclusion could be made that only the surface temperature of the emitter affected the radiative Nusselt number in geometrical and physical conditions under study.

It was interesting to note that an increase in the Rayleigh number slightly changed the convective Nusselt numbers at the bottom horizontal (Fig. $13 c$ ) and vertical (Fig. $13 d$ ) fluid-solid interfaces. At the same time, the $N u_{\text {rad }}$ was increased approximately four times in a range of $6 \cdot 10^{4} \leq R a \leq 4 \cdot 10^{6}$. It should be noted that an increase in the radiative Nusselt number was not directly related to an enhancement of the natural convection process. When thermophysical properties and temperature difference were constant, buoyancy force was increased due to a change in the characteristic size of the cavity. A reasonable conclusion could be made that an increase in the Rayleigh number led to a rise in the conduction-radiation number. As a result, the radiative Nusselt number was increased. A similar pattern was observed for dependence of the mean Nusselt number versus surface emissivity (Fig. $13 e, f$ ). The $N u_{\text {rad }}$ was increased more than five times at the bottom horizontal fluid-solid interface in a range of $0.2 \leq \varepsilon \leq 0.8$. Obviously, a rise in the emissivity led to an enhancement of radiative heat transfer. As a result, radiative fluxes were increased. The fluid-solid interfaces started to absorb and, hence, emit more energy. The radiative Nusselt number was increased due to a rise in the radiative heat transfer rate.

As might be expected, an increase in the emitter length led to a rise in the radiative Nusselt number both at the bottom horizontal (Fig. $13 \mathrm{~g}$ ) and vertical (Fig. $13 h$ ) solid-fluid interfaces. This, at first sight, obvious regularity was associated with an enhancement of the radiant heat exchange due to an increase in the size of the radiant energy source. However, the radiative Nusselt number was approximately increased 3 times when $D$ was raised 10 times. Along with that the convective Nusselt number was slightly altered. 

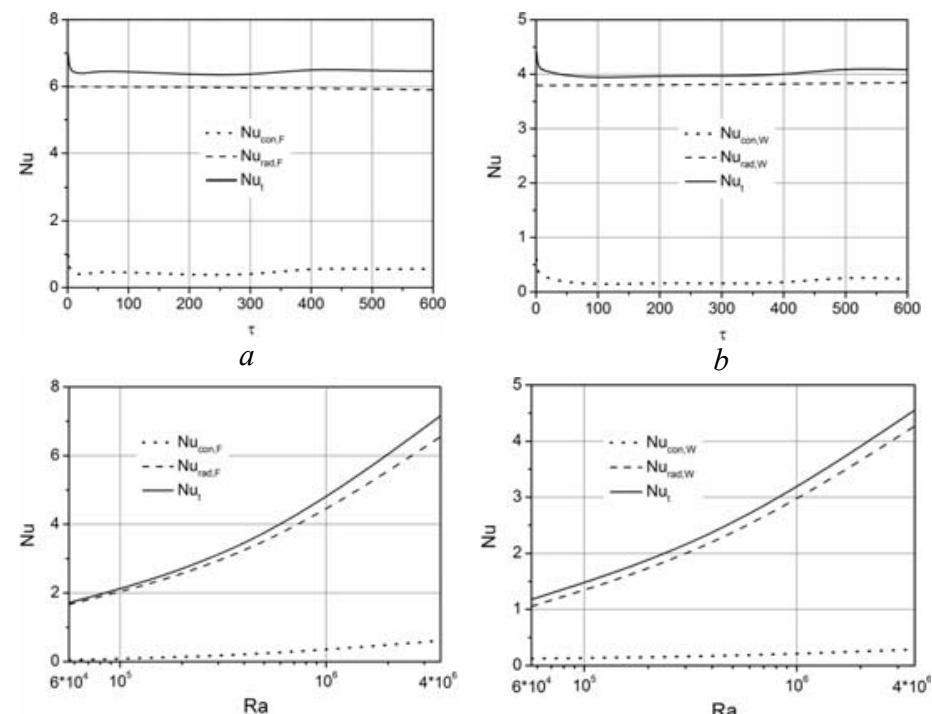

$c$

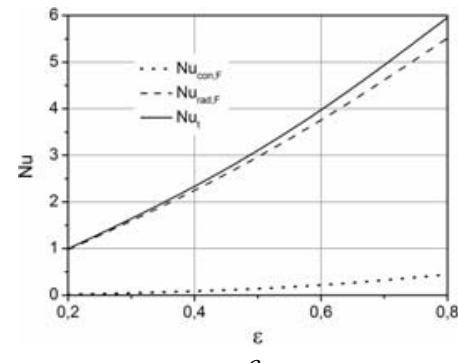

$e$

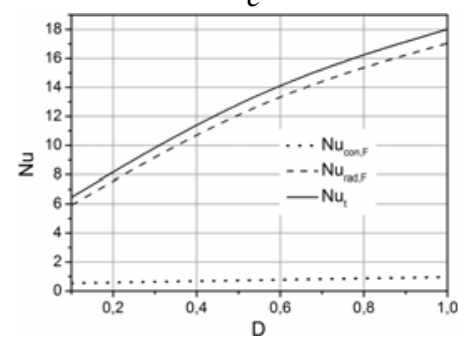

$g$
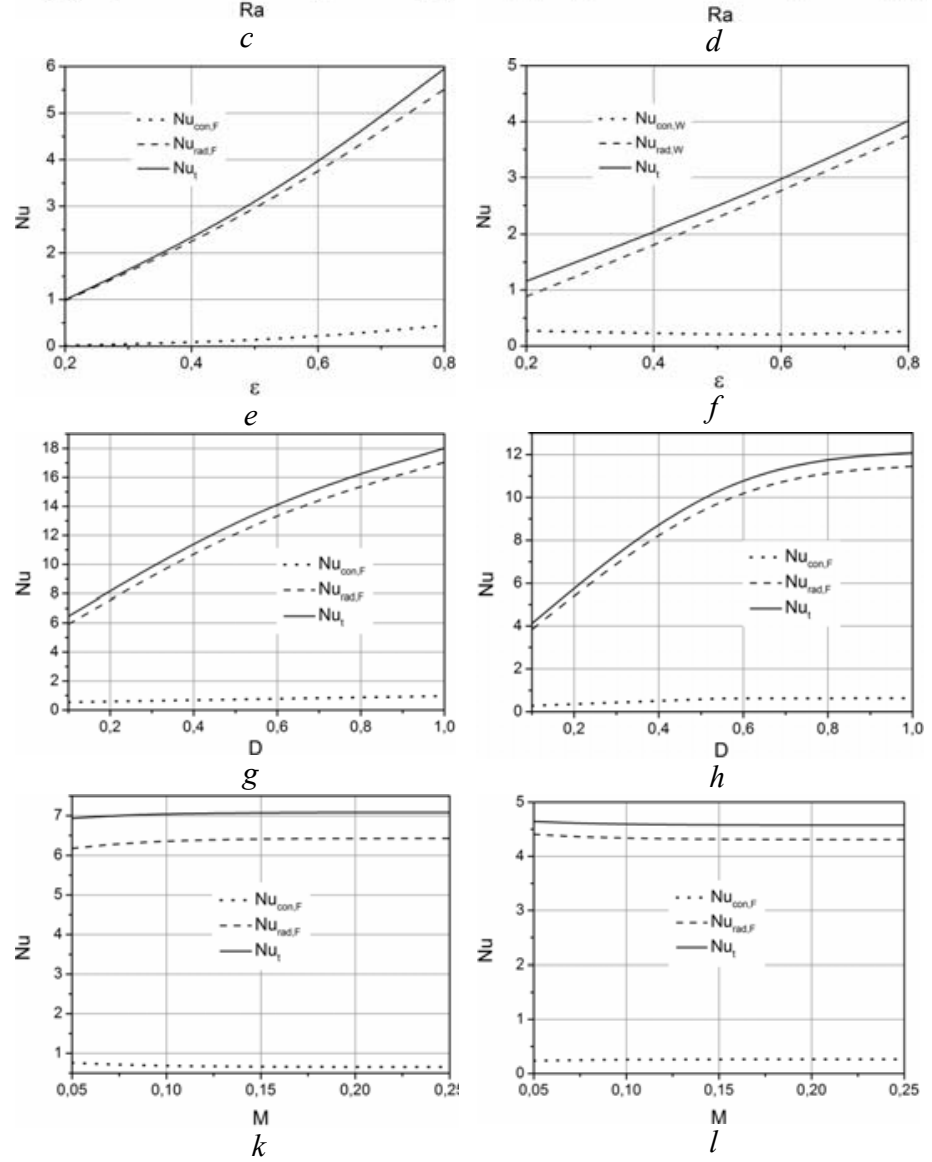

Fig. 13 Variation of the mean Nusselt numbers at the: $a, c, e, g, k-$ bottom horizontal fluid-solid interface; $b, d, f, h, l$-vertical fluid-solid interface

In subsection 3.5, it was found that an increase in the walls thickness in a range of $0.05 \leq M \leq 0.1$ led to a significant reduce in the temperature of the air cavity. And, therefore, it was interesting to note that integral characteristics of complex heat transfer was slightly altered (Fig. $13 k, l$ ). A similar regularity was also revealed in (Nouanegue et. al., 2009). In accordance with the mathematical modelling results, Nouanegue et. al. (2009) concluded that the effect of conductivity ratio and wall thickness on the Nusselt numbers was not significant. Such consequence was partly proved in this study.

\section{CONCLUSIONS}

Unsteady heat transfer process via conduction, laminar natural convection, and surface radiation was numerically investigated in a closed rectangular cavity with radiant heating source. The main findings are as follows:

- An increase in the dimensionless time in a range of $5 \leq \tau \leq 600$ led to a rise in the temperature of the fluid-solid interfaces approximately five times. However, the convective Nusselt number was slightly changed with time.

- When the Rayleigh number was varied from $6 \cdot 10^{4}$ to $4 \cdot 10^{6}$, the radiative Nusselt number was approximately increased four times. However, this factor was not associated with the enhancement of the natural convection. When $\mathrm{Ra}$ was increased, both the characteristic size of the cavity and conduction-radiation number were also risen. Along with that only two symmetrical large-scale convective cells were formed in the solution domain in a range of $R a$ under study.

- When the emissivity of the fluid-solid interfaces was $\varepsilon \leq 0.6$, thermal stratification was observed in the top half of the cavity due to the predominance of conduction near the radiant heating source. An increase in the surface emissivity four times led to a rise in the $N u_{\text {rad }, F}$ approximately 5.5 times.

Along with that $N u_{r a d, W}$ was approximately increased four times.

- An increase in the emitter length in a range of $0.1 \leq D \leq 1$ led to a rise in the temperature of the solution domain and modification of the flow pattern. However, with a tenfold increase of $D$ the radiative Nusselt number was increased approximately 3 times both at the bottom horizontal and vertical solid-fluid interfaces.

- In was found that the effect of walls thickness on integral characteristics of complex heat transfer was not significant. However, at the same time, an increase in the $M$ led to a reduce in the temperature of the air cavity.

- According to the results of the integral analysis, it was found that the convective Nusselt number was slightly changed in geometrical and physical conditions under study. A reasonable conclusion could be made that the radiative parameters (emissivity, view factors, and conductionradiation number) of the system under study predominantly affected the formation of the differential and integral conjugate heat transfer characteristics.

Further studies can include the analysis of the conjugate heat transfer under the operating conditions of a non-isothermal radiant heating source, turbulent flow regime and three-dimensional statement of the problem.

\section{ACKNOWLEDGEMENTS}

The research is carried out at Tomsk Polytechnic University within the framework of Tomsk Polytechnic University Competitiveness Enhancement Program.

\section{NOMENCLATURE}

$B$

length of the emitter, $\mathrm{m}$

$D \quad$ dimensionless length of the emitter

$F \quad$ view factor

Fo Fourier number

$g \quad$ gravitational acceleration, $\left(\mathrm{m} / \mathrm{s}^{2}\right)$

$J \quad$ dimensionless radiosity

$l \quad$ thickness of the walls

$L \quad$ characteristic length of the cavity (m) 


$\begin{array}{ll}M & \text { dimensionless thickness the walls } \\ n & \text { normal to a surface } \\ N_{r} & \text { conduction-radiation parameter } \\ \mathrm{Nu} & \text { mean Nusselt number } \\ P r & \text { Prandtl number } \\ Q_{r} & \text { dimensionless irradiation } \\ R a & \text { Rayleigh number } \\ t & \text { time (s) } \\ t_{0} & \text { time scale (s) } \\ T_{0} & \text { initial temperature }(\mathrm{K}) \\ T_{h} & \text { surface temperature of the emitter }(\mathrm{K}) \\ V_{0} & \text { velocity scale }(\mathrm{m} / \mathrm{s}) \\ X, Y & \text { coordinates }(\mathrm{m})\end{array}$

\section{Greek Symbols}

$\begin{array}{ll}\beta & \text { coefficient of thermal expansion }\left(\mathrm{K}^{-1}\right) \\ \varepsilon & \text { surface emissivity } \\ \Theta & \text { dimensionless temperature } \\ \tau & \text { dimensionless time } \\ \chi & \text { temperature ratio } \\ \psi & \text { stream function }\left(\mathrm{m}^{3} / \mathrm{s}\right) \\ \psi_{0} & \text { scale of stream function }\left(\mathrm{m}^{3} / \mathrm{s}\right) \\ \Psi & \text { dimensionless stream function analogue } \\ \omega & \text { vorticity }\left(\mathrm{s}^{-1}\right) \\ \omega_{0} & \text { scale of vorticity }\left(\mathrm{s}^{-1}\right) \\ \Omega & \text { dimensionless analogue of vorticity } \\ \text { Subscripts } & \\ 1 & \text { fluid } \\ 2 & \text { solid } \\ 3 & \text { heater }\end{array}$

\section{REFERENCES}

Ait-taleb, T., Abdelbaki, A. and Zrikem, Z., 2008, "Numerical Simulation of Coupled Heat Transfers by Conduction, Natural Convection and Radiation in Hollow Structures Heated from Below or Above," International Journal of Thermal Sciences, 47 (4), 378-387. https://doi.org/10.1016/j.ijthermalsci.2007.01.035

Akiyama, M. and Chong, Q.P., 1997, "Numerical Analysis of Natural Convection with Surface Radiation in a Square Enclosure," Numerical Heat Transfer; Part A: Applications, 32 (4), 419-433.

http://dx.doi.org/10.1080/10407789708913899

Anil Kumar Sharma, Velusamy, K. and Balaji, C., 2008, "Interaction of Turbulent Natural Convection and Surface Thermal Radiation in Inclined Square Enclosures," Heat and Mass Transfer, 44 (10), 11531170.

https://doi.org/10.1007/s00231-007-0353-5

Asako, Y. and Nakamura, H., 1982, "Heat Transfer Across a Parallelogram Shaped Enclosure," Bulletin of JSME, 25, 1412-1424.

Ashish Gad, M. and Balaji, C., 2010, "Effect of Surface Radiation on RBC in Cavities Heated from Below," International Communications in Heat and Mass Transfer, 37, 1459-1464.

https://doi.org/10.1016/j.icheatmasstransfer.2010.08.003

Aydin, O., 1999, "Transient Natural Convection in Rectangular Enclosures Heated from One Side and Cooled from Above," International Communications in Heat and Mass Transfer, 26, 135144.

https://doi.org/10.1016/S0735-1933(98)00129-8
Bae, J.H. and Hyun, J.M., 2004, "Time-Dependent Buoyant Convection in an Enclosure with Discrete Heat Sources," International Journal of Thermal Sciences, 43, 3-11. https://doi.org/10.1016/S1290-0729(03)00102-9

Baek, S.-W. and Kim, T.-Y., 2002, "Effects of Surface Radiation on the Unsteady Natural Convection in a Rectangular Enclosures," KSAS International Journal, 3 (1), 95-104.

Bouafia, M., Hamimid, S. and Guellal, M., 2015, "Non-Boussinesq Convection in a Square Cavity with Surface Thermal Radiation," International Journal of Thermal Sciences, 96, 236-247. https://doi.org/10.1016/j.ijthermalsci.2015.04.017

Cherifi, M., Laouar-Meftah, S., Benbrik, A., Lemonnier, D. and Saury, D., 2015, "Interaction of Radiation with Double-Diffusive Natural Convection in a Three-Dimensional Cubic Cavity Filled with a NonGray Gas Mixture in Cooperating Cases," Numerical Heat Transfer; Part A: Applications, 69 (5), 479-496. http://dx.doi.org/10.1080/10407782.2015.1090233

Codina, R., 2001, “A Stabilized Finite Element Method for Generalized Stationary Incompressible Flows, Computer Methods in Applied Mechanics and Engineering," 190 (20-21), 2681-2706.

http://dx.doi.org/10.1016/S0045-7825(00)00260-7

de Vahl Davis, G., 1983, "Natural Convection of Air in a Square Cavity: a Benchmark Numerical Solution," International Journal for Numerical Methods in Fluids, 3 (3), 249-264.

http://dx.doi.org/10.1002/fld.1650030305

Dixit, H.N. and Babu, V., 2006, "Simulation of High Rayleigh Number Natural Convection in a Square Cavity Using the Lattice Boltzmann Method," International Journal of Heat and Mass Transfer, 49 (3-4), 727-739.

https://doi.org/10.1016/j.ijheatmasstransfer.2005.07.046

Hamimid, S., Guellal, M. and Bouafia M., 2016, "Numerical Study of Natural Convection in a Square Cavity under Non-Boussinesq Conditions," Thermal Science, 20 (5), 1509-1517. http://dx.doi.org/10.2298/TSCI130810084H

Hinojosa, J.F., Buentello, D., Xamán, J. and Pérez-Tello, M., 2017, "The Effect of Surface Thermal Radiation on Entropy Generation in an Open Cavity with Natural Convection," International Communications in Heat and Mass Transfer, 8, 164-174.

https://doi.org/10.1016/j.icheatmasstransfer.2016.12.018

Hottel, H.C. and Sarofim, A.F., 1967, Radiative Transfer. McGrawHill, New York.

Jani S., Mahmoodi M., Amini M. and Jam J.E., 2014, "Numerical Investigation of Natural Convection Heat Transfer in a Symmetrically Cooled Square Cavity with a Thin Fin on its Bottom Wall," Thermal Science, 18 (4), 1119-1132.

http://dx.doi.org/10.2298/TSCI110612139J

Kuznetsov, G.V. and Sheremet, M.A., 2009, "Conjugate Natural Convection with Radiation in an Enclosure," International Journal of Heat and Mass Transfer, 52 (9-10), 2215-2223.

https://doi.org/10.1016/j.ijheatmasstransfer.2008.12.006

Kuznetsov, G.V. and Sheremet, M.A., 2010, "Numerical Simulation of Turbulent Natural Convection in a Rectangular Enclosure Having Finite Thickness Walls," International Journal of Heat and Mass Transfer, 53 (1-3), 163-177.

https://doi.org/10.1016/j.ijheatmasstransfer.2009.09.043

Kuznetsov, G.V. and Sheremet, M.A., 2011, "A Numerical Simulation of Double-Diffusive Conjugate Natural Convection in an Enclosure," International Journal of Thermal Sciences, 50 (10), 1878-1886. https://doi.org/10.1016/j.ijthermalsci.2011.05.003 
Markatos, N.C. and Pericleous, K.A., 1984, "Laminar and Turbulent Natural Convection in an Enclosed Cavity," International Journal of Heat and Mass Transfer, 27 (5), 755-772.

https://doi.org/10.1016/0017-9310(84)90145-5

Martyushev, S. G. and Sheremet, M. A., 2014a, "Conjugate Natural Convection Combined with Surface Thermal Radiation in a ThreeDimensional Enclosure with a Heat Source," International Journal of Heat and Mass Transfer, 73, 340-353.

https://doi.org/10.1016/j.ijheatmasstransfer.2014.02.009

Martyushev, S. G. and Sheremet, M. A., 2014b, "Conjugate Natural Convection Combined with Surface Thermal Radiation in an Air Filled Cavity with Internal Heat Source," International Journal of Thermal Sciences, 76, 51-67.

https://doi.org/10.1016/j.ijthermalsci.2013.08.012

Mezrhab, A, Amraqui, S. and Abid, C., 2010, "Modelling of Combined Surface Radiation and Natural Convection in a Vented "T" Form Cavity," International Journal of Heat and Fluid Flow, 31 (1), 83-92. https://doi.org/10.1016/j.ijheatfluidflow.2009.10.004

Nouanegue, H., Muftuoglu. A. and Bilgen, E., 2008, "Conjugate Heat Transfer by Natural Convection, Conduction and Radiation in Open Cavities," International Journal of Heat and Mass Transfer, 51 (25-26), 6054-6062.

https://doi.org/10.1016/j.ijheatmasstransfer.2008.05.009

Nouanegue, H., Muftuoglu, A. and Bilgen E., 2009, "Heat Transfer by Natural Convection, Conduction and Radiation in an Inclined Square Enclosure Bounded with a Solid Wall," International Journal of Thermal Sciences, 48 (5), 871-880.

https://doi.org/10.1016/j.ijthermalsci.2008.06.008

Rajkumar, M. R., Venugopal, G. and Anil Lal, S., 2011, "Natural Convection with Surface Radiation from a Planar Heat Generating Element Mounted Freely in a Vertical Channel," Heat and Mass Transfer, 47(7), 789-805. https://doi.org/10.1007/s00231-010-0734-z

Roache, P. J., 1976, Computational Fluid Dynamics, Hermosa Publishers, Albuquerque.
Sadin, R., Chegini, G.-R. and Sadin, H., 2014, "The Effect of Temperature and Slice Thickness on Drying Kinetics Tomato in the Infrared Dryer," Heat and Mass Transfer, 50 (4), 501 - 507. https://doi.org/10.1007/s00231-013-1255-3

Suslov, S. A. and Paolucci, S., 1997, "Nonlinear Analysis of Convection Flow in a Tall Vertical Enclosure under non-Boussinesq Conditions," Journal of Fluid Mechanics, 344, 1-41.

Samarskii, A.A., 1977, The Theory of Difference Schemes [in Russian], Nauka, Moscow.

Vierendeels, J., Merci, B. and Dick, E., 2004, “A Multigrid Method for Natural Convective Heat Transfer with Large Temperature Differences," Journal of Computational and Applied Mathematics, 168 (1-2), 509-517.

http://dx.doi.org/10.1016/j.cam.2003.08.081

Wang, H., Xin, S. and Le Quere P., 2006, "Etude Numerique du Couplage de la Convection Naturelle Avec le Rayonnement de Surfaces en Cavite Carree Remplie D'air," Comptes Rendus - Mecanique, 334 (1), 48-57.

https://doi.org/10.1016/j.crme.2005.10.011

Zhong Z.Y., Yang K.T. and Lloyd J.R., 1985, "Variable Property Effects in Laminar Natural Convection in a Square Enclosure," Journal of Heat transfer, 107 (1), 133-138.

http://dx.doi.org/10.1115/1.3247369

Xin, S., Salat, J., Joubert, P., Sergent, A., Penot, F. and Le Quéré, P., 2013, "Resolving the Stratification Discrepancy of Turbulent Natural Convection in Differentially Heated Air-Filled Cavities. Part III: A full convection-conduction-surface radiation coupling," International Journal of Heat and Fluid Flow, 42, 33-48. https://doi.org/10.1016/j.ijheatfluidflow.2013.01.021

Zhu, Z.J. and Yang, H.X., 2003, "Numerical Investigation of Transient Laminar Natural Convection of Air in a Tall Cavity," Heat and Mass Transfer, 39, 579-587.

https://doi.org/10.1007/s00231-002-0385-9 